\title{
ARTICLES
}

\section{Many-body interaction analysis: Algorithm development and application to large molecular clusters}

\author{
Anant D. Kulkarni, V. Ganesh, and Shridhar R. Gadre ${ }^{\text {a) }}$ \\ Department of Chemistry, University of Pune, Pune 411 007, India
}

(Received 1 April 2004; accepted 16 June 2004)

\begin{abstract}
A completely automated algorithm for performing many-body interaction energy analysis of clusters (MBAC) [M. J. Elrodt and R. J. Saykally, Chem. Rev. 94, 1975 (1994); S. S. Xantheas, J. Chem. Phys. 104, 8821 (1996)] at restricted Hartree-Fock (RHF)/M $\widetilde{A}$ Plesset 2nd order perturbation theory (MP2)/density functional theory (DFT) level of theory is reported. Use of superior guess density matrices (DM's) for smaller fragments generated from DM of the parent system and elimination of energetically insignificant higher-body combinations, leads to a more efficient performance (speed-up up to 2) compared to the conventional procedure. MBAC approach has been tested out on several large-sized weakly bound molecular clusters such as $\left(\mathrm{H}_{2} \mathrm{O}\right)_{n}, n=8,12,16,20$ and hydrated clusters of amides and aldehydes. The MBAC results indicate that the amides interact more strongly with water than aldehydes in these clusters. It also reconfirms minimization of the basis set superposition error for large cluster on using superior quality basis set. In case of larger weakly bound clusters, the contributions higher than four body are found to be repulsive in nature and smaller in magnitude. The reason for this may be attributed to the increased random orientations of the interacting molecules separated from each other by large distances. (C) 2004 American Institute of Physics. [DOI: 10.1063/1.1780156]
\end{abstract}

\section{INTRODUCTION}

Investigation of many-body interactions is considered to be of prime importance in experimental as well as theoretical studies of molecular clusters. ${ }^{1,2}$ This importance may be attributed to the insights provided by many-body interaction analysis (MBA) for understanding the nature of binary interactions and the cooperativity in such intermolecular clusters. Also, as stated by Elrod and Saykally, ${ }^{1}$ the many-body effects play a critical role in determining the most important physical properties.

MBA studies are in practice for the past 50 years or more. Axilrod and Teller ${ }^{2}$ have employed third order perturbation theory for calculating three-body triple-dipole dispersion energy for rare gas atoms. Later on, Rosen ${ }^{3}$ employed the valence bond approach for calculating short-range energy of linear and triangular geometries of helium atoms and determined functional forms for the three-body interaction for these geometries. Shostak ${ }^{4}$ studied the nonadditivity in linear $\mathrm{He}_{3}$ system by using LCAO-MO approach. Jansen ${ }^{5}$ extended MBA approach by introducing a new model and simplifying the calculation of many-body terms for rare gases. Lombardi and Jansen ${ }^{6}$ proposed further extension of this approach up to four-body effects and noticed that in the case of most relevant geometries of crystal structures these effects are negligible. Curiously, many of the earlier MBA-related studies were limited to rare gas clusters.

\footnotetext{
a) Author to whom correspondence should be addressed. Electronic mail: gadre@chem.unipune.ernet.in
}

The earlier results of MBA approach for the molecular clusters of $\left(\mathrm{H}_{2} \mathrm{O}\right)_{2-3}$ were reported by Hankins and co-workers. ${ }^{7}$ They investigated the potential for pairs and triplets of water molecules and highlighted the need to carry out the calculation for water tetramers. In the recent years, the importance of MBA for studying molecular clusters has been stressed by Xantheas, ${ }^{8}$ in light of its potential for development of new, and the evaluation of the existing, interaction potentials for water. He also proposed the need to explore extensive regions of the potential energy surface to parametrize the potentials from fits to $a b$ initio calculations. Xantheas and co-workers ${ }^{9,10}$ have employed this concept extensively to study the many-body effects in various water clusters, $\left(\mathrm{H}_{2} \mathrm{O}\right)_{n}, n=2-6$ and acetylene..(water) $)_{n}, n$ $=1-4$. On the basis of many-body interaction analysis, they have suggested that the global and the low-lying ring networks are stabilized by the maximum of the many-body (mainly three-body) terms, whereas the higher-lying minima are mainly described by two-body interactions. Recently, Kulkarni et al. ${ }^{11}$ have also applied MBA to the clusters of hydrogen peroxide at MP2 level of theory.

Although conceptually MBA is of vital importance from theoretical as well as experimental points of view, the reports on MBA of large molecular clusters are still rather scarce in the literature. ${ }^{1}$ To the best of the authors' knowledge, the largest system studied with this approach, until now, is water hexamer ${ }^{8}$ at HF/aug-cc- $p$-VDZ level of theory. Performing this analysis manually is an extremely arduous task, especially with an increase in the number of total molecules in the given cluster. Application of basis set superposition error 
(BSSE) correction to many-body energy terms has been discussed by various researchers. ${ }^{12-15}$ White and Davidson ${ }^{13}$ have performed the study on hydrogen bond in ice where the binding energy of the ice cluster is represented as a sum of two-body and three-body terms. The energy of each body term is again decomposed into its constituents according to Morokuma energy decomposition scheme. ${ }^{14}$ According to their study, ${ }^{13}$ the major contribution to binding energy comes from two-body terms, although the three-body terms are also essential to reproduce the binding energy of the cluster. The generalization of Boys-Bernardi scheme $\mathrm{e}^{12}$ has been proposed by Valiron and Mayer. ${ }^{15}$ This method involves the computation of BSSE-corrected energy terms in a recursive manner using the hierarchy of one-, two-, three-body, etc., energy terms, and the recomputation of each such contribution in basis sets of subclusters of increasing size. Their results for $(\mathrm{He})_{3}$ suggest that properly BSSE-corrected cluster calculations may combine refined two-body interactions with approximate many-body corrections. Recently, Mierzwicki and Latjka $^{16}$ have proposed yet another modification of BoysBernardi scheme, enabling it to perform BSSE for $N$-body clusters. The validity of this new scheme has been tested for $(\mathrm{HF})_{n}, n=3$ and 4 . A comparison of these results with the other counterpoise methods has also been presented by them. However, it should be noted that all these discussions pertain to only the small clusters with $n<5$. If one is interested in performing BSSE correction ${ }^{12-15}$ to the individual $n$-body energy terms for a medium or large molecular clusters (say $n>8$ ), then overall procedure becomes extremely tedious.

In view of this, the development of an efficient algorithm alongwith a fully automated code to carry out MBA, with or without BSSE corrections and benchmarking its performance as well as applications to a variety of weakly bound molecular clusters, has been felt worthwhile.

The present paper deals with the development of an automated code, many-body analysis of clusters (MBAC) to perform many-body analysis, in a generalized manner, of molecular clusters at ab initio level. The algorithm MBAC can also be employed to study BSSE correction to individual $n$-body terms as well as the entire molecular system. An important feature of this algorithm is the use of density matrix (DM) of parent system to obtain synthesized DM's ${ }^{17}$ for various molecular combinations and employing them as guess-DM's in the SCF procedure leading to time advantage over the conventional calculations. Presented below is the methodology of MBAC, followed by test applications to large molecular clusters.

\section{METHODOLOGY}

\section{A. Many-body analysis scheme (Refs. 1, 7, 8, and 10)}

The total energy $E_{n}$ of the $n$-body cluster (body: molecule) can be written as the sum of the one-, two-, three-, four-,,..,$n$-body terms according to

$$
E_{n}=E(1234 \ldots n),
$$

$$
\begin{aligned}
E_{n} \equiv & \sum_{i=1}^{n} E(i)+\sum_{i=1}^{n-1} \sum_{j>i}^{n} \Delta^{2} E(i j) \\
& (\text { one body) } \quad \text { (two body) } \\
& +\sum_{i=1}^{n=2} \sum_{j>i}^{n-1} \sum_{k>j}^{n} \Delta^{3} E(i j k) \\
& (\text { three body) } \\
& +\sum_{i=1} \sum_{j>i} \sum_{k>j} \sum_{l>k} \Delta^{4} E(i j k l)+\cdots \\
& (\text { four body }) \\
+ & \Delta^{n} E(1234 \ldots n) . \\
& (n \text { body })
\end{aligned}
$$

In expression (2), $E(i)$ are the energies of relaxed molecules in the cluster and $E(i j), E(i j k) \ldots$ are the energies of dimers, trimers, etc. The individual two-, three-, four-, $, \ldots, n-$ body terms are defined as follows:

$$
\begin{aligned}
\Delta^{2} E(i j)=E & (i j)-\{E(i)+E(j)\}, \\
\Delta^{3} E(i j k)= & E(i j k)-\{E(i)+E(j)+E(k)\}-\left\{\Delta^{2} E(i j)\right. \\
& \left.+\Delta^{2} E(i k)+\Delta^{2} E(j k)\right\}, \\
\Delta^{4} E(i j k l)= & E(i j k l)-\{E(i)+E(j)+E(k)+E(l)\} \\
& -\left\{\Delta^{2} E(i j)+\Delta^{2} E(i k)+\Delta^{2} E(i l)\right. \\
& \left.+\Delta^{2} E(j k)+\Delta^{2} E(j l)+\Delta^{2} E(k l)\right\} \\
& -\left\{\Delta^{3} E(i j k)+\Delta^{3} E(i j l)+\Delta^{3} E(i k l)\right. \\
& \left.+\Delta^{3} E(j k l)\right\},
\end{aligned}
$$

and so on. The binding energy of the $n$-body cluster is $\mathrm{BE}_{n}$ $=E_{n}-n E_{i}$, where $E_{i}$ is the energy of the constituent molecules. In case of heteroclusters, this expression has the form $\mathrm{BE}_{n}=E_{n}-x E_{i}-y E_{j}$, with $x$ and $y$ being the number of $i$ and $j$ constituents, respectively, and $x+y=n$.

The term involving one-body term $E_{R}=\sum_{i}^{n} E(i)-n E_{i}$ can be considered as the total relaxation energy $E_{R}$ of the constituent molecules in the cluster with respect to the gas phase monomeric units. This energy is a measure of the degree of strain that drives the constituent molecules in the cluster. $^{8}$ The average relaxation energy per molecule is then $E_{R} / n$.

The above equations are applicable to the cases of homoclusters as well as heteroclusters. However, in the latter case, the many-body terms can be grouped into two categories, viz., the terms containing the reference heteromolecule and those not containing heteromolecule.

From above discussion, the general expression for any $r$-body term can be written as

$$
\Delta^{r} E(i j \ldots r)=E(i j \ldots r)-\sum_{i=r-1}^{1} \Delta^{i} E(i j \ldots r),
$$

where the second term inside the summation is again expanded as the original equation which is composed of various combinations occurring due to all $i-1$ body terms (to be solved recursively). 


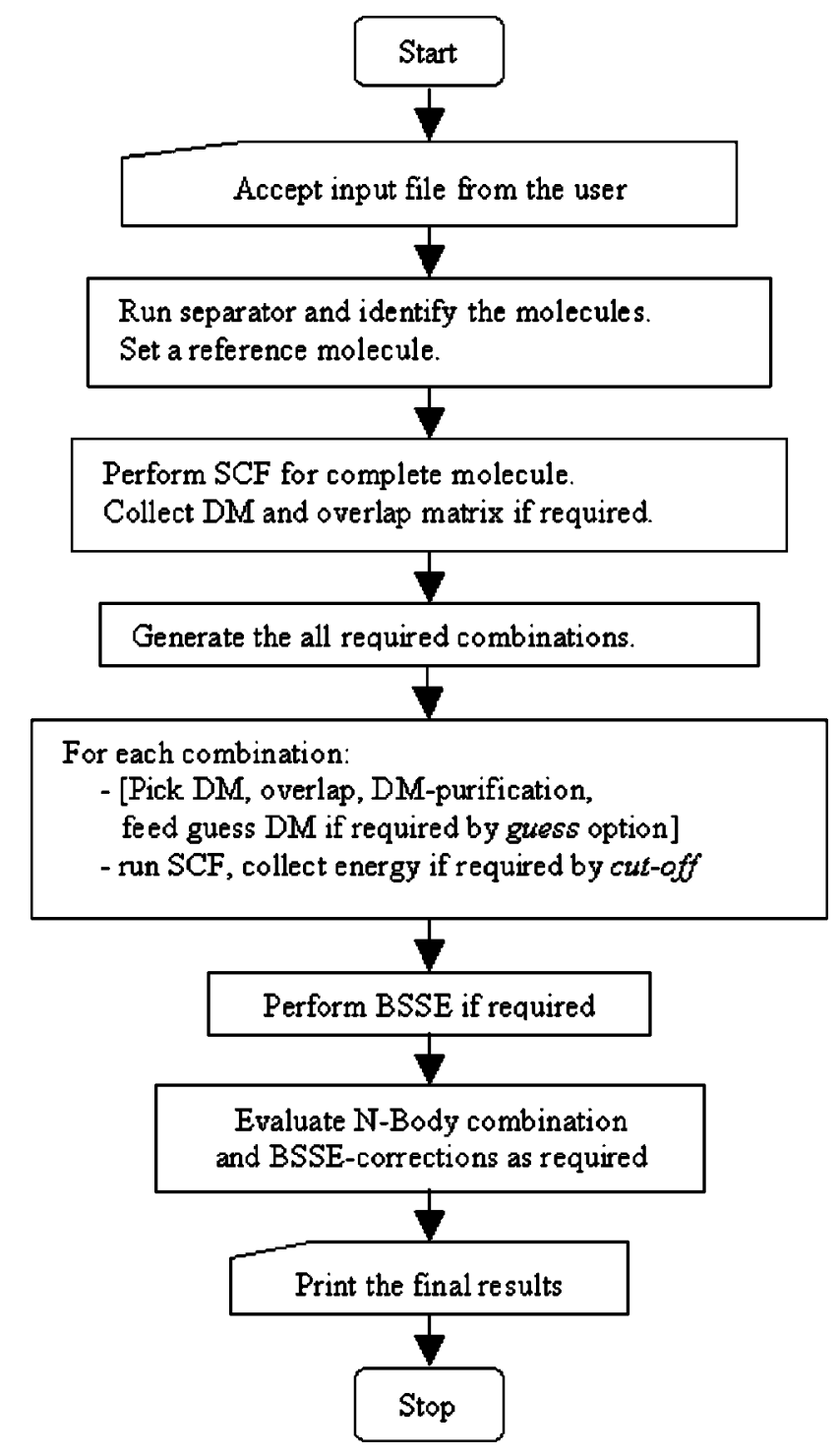

FIG. 1. Flowchart for many-body analysis of clusters (MBAC) algorithm. See text for details.

\section{B. Implementation of algorithm}

MBAC algorithm is exclusively designed to perform MBA on neutral molecular clusters. It can also be employed to perform BSSE corrections to individual body terms as well as a standalone algorithm to calculate BSSE correction to entire molecular system in an efficient and automated manner. The basic flow diagram of the program is presented in Fig. 1. The entire code has been written in FORTRAN 90 and compiled on GNU/Linux [Ref. 18(a)] using a highperforming compiler (unsupported version) from Intel. ${ }^{18(b)}$

The MBAC algorithm accepts input in Cartesian coordinates along with a set of options to be specified by the user (e.g., up to which body analysis is required, whether BSSE correction needs to be performed, etc.). Various molecules in the cluster are then identified for further processing. In order to apply this approach to heteromolecular systems, an option to define the reference molecule has been employed in the code. This option facilitates to understand various interactions in a given cluster in a detailed manner.
A single point energy calculation on the entire cluster is then performed by using standard $a b$ initio packages, ${ }^{19,20}$ though the program can be trivially modified for using in conjunction with any ab initio package. The guess option implemented in the program (to be used only with locally modified version of GAMESS) uses the DM modification technique from molecular tailoring approach (MTA) (Ref. 17) developed in our laboratory. This procedure involves obtaining the DM of parent molecular system at the user-requested level and basis set. Depending on the molecular combinations to be evaluated, parts of the DM and the overlap matrix (obtained previously from parent supermolecular cluster) are picked and McWeeny refinement scheme ${ }^{21}$ from MTA approach ${ }^{17}$ is performed. The resultant, refined DM forms an excellent starting guess for SCF calculation of appropriate combination. Also, a criterion (cutoff option) to perform a systematic elimination of energetically insignificant higherbody (four body onwards) terms has been implemented in the code. Before proceeding to the actual evaluation of $r$-body terms a cutoff criterion as $X \%$ (typical value used is $1 \%)$ of the absolute maximum of $(r-1)$-body terms is determined. If all the $(r-1)$ body terms constituting the particular $r$-body term are less than this cutoff, then the evaluation of this term is skipped and energy for this term is taken as zero. This criterion works effectively when the $r$-body terms (when $r>3$ ) in the large cluster are far-off from each other. It should also be noted that this cutoff does not result into any significant error in $r$-body energy term.

After evaluation of single point energies for required combinations, the program can compute BSSE corrections ${ }^{12}$ to individual terms. The procedure implemented for BSSE calculations is based on the schemes provided by the respective $a b$ initio packages. ${ }^{19,20}$ Here, the energy of each monomeric unit is recalculated by putting ghost orbitals on the other constituents in an automated and efficient way. A final summary of all the details and computations is stored into a file on disk.

\section{RESULTS AND DISCUSSION}

As pointed out in the Introduction, performing MBA for large-sized molecular clusters is an extremely arduous task. Consider a molecular cluster involving $n$ molecules. The total number of $r$-body terms to be evaluated is ${ }^{n} C_{r}$, where the total terms will be $2^{n}-1$ with ${ }^{n} C_{2}=n(n-1) /(2 !)$ and ${ }^{n} C_{3}$ $=n(n-1)(n-2) /(3$ !) being the number of two- and threebody terms, respectively. ${ }^{22}$ Also, if one is interested in BSSE correction to these individual terms, then the problem become further complicated and time consuming.

The results of completely automated program (MBAC) to perform such an analysis are summarized below. The program performs single point energy calculations at the user specified level/basis set, from an external $a b$ initio package. ${ }^{19,20}$ The speciality of our approach is the use of a locally modified version of GAMESS along with (DM) modification technique, ${ }^{17}$ which allows to obtain the DM and the overlap matrix along with energy of the parent cluster. This is a very vital step, ensuring faster SCF convergence for the molecular combinations that need to be evaluated. 
TABLE I. MBAC-code performance analysis for various molecular clusters at RHF and MP2 levels using 6-31G(d,p) basis set. The timings (in min) are given for many-body calculations only upto four-body calculations. All the calculations have been performed on P-IV, $2.8 \mathrm{GHz}, 1 \mathrm{~GB}$ DDRAM, Linux. See text for details. I, timing and energy terms with guess and $1 \%$ cutoff option; II, timing and energy terms without guess and cutoff option.

\begin{tabular}{llrr}
\hline \hline Mol. cluster & Level & I (min) & II (min) \\
\hline$\left(\mathrm{H}_{2} \mathrm{O}\right)_{12}$ & RHF & 64.2 & 95.0 \\
$\left(\mathrm{H}_{2} \mathrm{O}\right)_{16}$ & RHF & 185.6 & 280.1 \\
$\left(\mathrm{H}_{2} \mathrm{O}\right)_{20}$ & RHF & 416.1 & 617.3 \\
$\left(\mathrm{H}_{2} \mathrm{O}\right)_{8}$ & MP2 & 23.2 & 33.3 \\
$\left(\mathrm{H}_{2} \mathrm{O}\right)_{12}$ & MP2 & 106.5 & 119.1 \\
$\mathrm{FM} \cdots\left(\mathrm{H}_{2} \mathrm{O}\right)_{12}$ & RHF & 137.3 & 197.3 \\
$\mathrm{FM} \cdots\left(\mathrm{H}_{2} \mathrm{O}\right)_{16}$ & RHF & 326.6 & 497.3 \\
$\mathrm{FM} \cdots\left(\mathrm{H}_{2} \mathrm{O}\right)_{24}$ & RHF & 1143.2 & 2081.1 \\
FM $\cdots\left(\mathrm{H}_{2} \mathrm{O}\right)_{28}$ & RHF & 1651.2 & 3301.5 \\
$\mathrm{AM} \cdots\left(\mathrm{H}_{2} \mathrm{O}\right)_{16}$ & RHF & 469.1 & 733.1 \\
FLD $\cdots\left(\mathrm{H}_{2} \mathrm{O}\right)_{16}$ & RHF & 264.9 & 401.5 \\
ALD $\cdots\left(\mathrm{H}_{2} \mathrm{O}\right)_{16}$ & RHF & 352.3 & 544.1 \\
\hline \hline
\end{tabular}

The program has been tested for several molecular systems including the water clusters, ${ }^{23}$ the hydrated clusters of formamide (FM), acetamide (AM), formaldehyde (FLD), and acetaldehyde (ALD) (Ref. 24) with various options and at RHF and MP2 levels of theory. The prototype hydrated clusters of formamide are depicted in Fig. 4.

\section{A. Efficiency of code}

The applicability of the present approach can be seen from the ability of MBAC algorithm to perform the analysis of $\left(\mathrm{H}_{2} \mathrm{O}\right)_{n}, n=8,12,16$, and 20 , in an efficient and automated manner. The calculations were restricted only up to four-body interaction energies since the contribution of the higher-body terms to the total interaction energy is found to be negligible for the large cluster. The total number of terms in $n=20$ case is 6195 (4845 four-body +1140 three-body +190 two-body +20 one-body terms). A manual calculation, sorting, and analysis would indeed be a massive and errorprone task.

The approach of providing the initial guess DM for SCF calculations as well as the skipping of numerically insignificant higher-body contributions, proposed in the MBAC algorithm, is seen to work efficiently for the large clusters ( $n$ $>12$ ), making the evaluation of many-body terms $\sim 1.4$ to 2.0 times faster than the traditional approach. The details of various benchmark timings are summarized in Table I, whereas the other details of energetics for homoclusters as well as heteroclusters are given in Tables II-VI.

TABLE II. MBAC-energy analysis for $\left(\mathrm{H}_{2} \mathrm{O}\right)_{n}, n=12,16$, and 20 at RHF/6-31G $(d, p)$ level. The calculations are performed only upto four-body calculations and energies are in kcal mol. ${ }^{-1}$ See text for details. $E_{\text {Contr }}$ denotes energy contributions.

\begin{tabular}{lrrr}
\hline \hline$E_{\text {Contr }}$ & $\left(\mathrm{H}_{2} \mathrm{O}\right)_{12}$ & $\left(\mathrm{H}_{2} \mathrm{O}\right)_{16}$ & $\left(\mathrm{H}_{2} \mathrm{O}\right)_{20}$ \\
\hline$E$ (two-body term) & -106.75 & -147.62 & -189.26 \\
$E$ (three-body term) & -15.97 & -21.63 & -27.32 \\
$E$ (four-body term) & -1.44 & -1.90 & -2.56 \\
$E_{R}$ & 1.64 & 2.14 & 2.57 \\
$\Delta E$ & -122.39 & -168.81 & -216.28 \\
\hline \hline
\end{tabular}

TABLE III. Details of BSSE analysis of $\left(\mathrm{H}_{2} \mathrm{O}\right)_{n}, n=12,16,20$ at RHF level using (A) 6-31G(d,p), (B) 6-31+G(d,p), and (C) 6-311+ $+G(2 d, 2 p)$ basis sets. All the energies are in $\mathrm{kcal} \mathrm{mol}^{-1}$. Raw $\Delta E$ denotes $\Delta E$ uncorrected for BSSE and $\Delta E_{\mathrm{BSSE}-\mathrm{CORR}}$ denotes $\Delta E$ corrected for BSSE.

\begin{tabular}{lcccc}
\hline \hline Cluster & Energies & A & B & C \\
\hline$\left(\mathrm{H}_{2} \mathrm{O}\right)_{12}$ & Raw $\Delta E$ & -122.39 & -99.53 & -82.79 \\
& $\Delta E_{\text {BSSE-CORR }}$ & -100.44 & -89.29 & -79.54 \\
& BSSE correction \% & 17.9 & 10.3 & 3.9 \\
$\left(\mathrm{H}_{2} \mathrm{O}\right)_{16}$ & Raw $\Delta E$ & -168.81 & -136.65 & -113.74 \\
& $\Delta E_{\text {BSSE-CORR }}$ & -137.88 & -122.29 & -108.99 \\
& BSSE correction \% & 18.3 & 10.5 & 4.2 \\
$\left(\mathrm{H}_{2} \mathrm{O}\right)_{20}$ & Raw $\Delta E$ & -216.28 & -174.71 & $\ldots$ \\
& $\Delta E_{\text {BSSE-CORR }}$ & -176.06 & -156.30 & $\cdots$ \\
& BSSE correction \% & 18.6 & 10.5 & $\cdots$ \\
\hline \hline
\end{tabular}

The MBAC performance for $\left(\mathrm{H}_{2} \mathrm{O}\right)_{8}$ and $\left(\mathrm{H}_{2} \mathrm{O}\right)_{12}$ (cf. Table IV) shows the applicability of the algorithm at perturbative level of theory. It may be noted that one gets the time advantage at MP2 level as well. However, this advantage is not substantial since the advantage due to guess option comes into picture only up to the calculation of RHF energy.

For heteroclusters of FM $\cdots\left(\mathrm{H}_{2} \mathrm{O}\right)_{n}, n=24$ and 28, up to four-body terms, the manual evaluation and analysis is indeed a formidable task. This is due to the large number of terms involved, viz., 15275 and 27840 for $n=24$ and 28, respectively. The analysis here has also been restricted up to four-body terms since the higher-body terms are negligible and form the nonadditive part of the interaction energy. The details of timings and energy analysis are given in Tables I and $\mathrm{V}$.

It may be seen that the MBAC analysis provides an effective approach to understand the nature of cooperativity in a weakly bound cluster at molecular level. The following section represents a discussion providing the physical interpretation of MBAC results when applied to various molecular clusters.

\section{B. Application of MBAC analysis}

\section{Homoclusters of water, $\left(\mathrm{H}_{2} \mathrm{O}\right)_{n}$}

It has been remarked by Xantheas ${ }^{9,10}$ that the positive percentage contribution of any $n$-body aggregate indicates that it is an additive or attractive contribution while the negative one indicates a repulsive or nonadditive contribution. In the present case of water clusters, total two-, three-, as well

TABLE IV. MBAC-energy analysis for $\left(\mathrm{H}_{2} \mathrm{O}\right)_{n}, n=8$ and 12 at MP2/6-31G $(d, p)$ level. The analysis is performed up to four-body calculations and energies are in $\mathrm{kcal} \mathrm{mol}^{-1}$. Notations and other details as in Table II. See text for details.

\begin{tabular}{lrr}
\hline \hline$E_{\text {Contr }}$ & $\left(\mathrm{H}_{2} \mathrm{O}\right)_{8}$ & $\left(\mathrm{H}_{2} \mathrm{O}\right)_{12}$ \\
\hline$E$ (two-body term) & -84.60 & -136.87 \\
$E$ (three-body term) & -19.54 & -18.05 \\
$E$ (four-body term) & -2.19 & -1.87 \\
$E_{R}$ & 2.24 & 2.88 \\
$\Delta E$ & -102.07 & -153.77 \\
\hline \hline
\end{tabular}


TABLE V. The energy contributions from MBAC analysis for FM $\cdots\left(\mathrm{H}_{2} \mathrm{O}\right)_{n}, n=12,16,24$, and 28 at RHF/6-31G( $\left.d, p\right)$ level. The calculations are performed only up to four-body calculations and energies are in $\mathrm{kcal} \mathrm{mol}{ }^{-1}$. See text for details.

\begin{tabular}{lcccc}
\hline \hline$E_{\text {Contr }}$ & $\mathrm{FM} \cdots\left(\mathrm{H}_{2} \mathrm{O}\right)_{12}$ & $\mathrm{FM} \cdots\left(\mathrm{H}_{2} \mathrm{O}\right)_{16}$ & $\mathrm{FM} \cdots\left(\mathrm{H}_{2} \mathrm{O}\right)_{24}$ & $\mathrm{FM} \cdots\left(\mathrm{H}_{2} \mathrm{O}\right)_{28}$ \\
\hline$E$ (two-body & -117.08 & -157.95 & -223.27 & -260.27 \\
term) & $(-106.22)$ & $(-147.06)$ & $(-185.96)$ & $(-224.64)$ \\
$E$ (three-body & -17.58 & -23.21 & -34.24 & -39.08 \\
term) & $(-15.56)$ & $(-21.23)$ & $(-24.80)$ & $(-31.29)$ \\
$E$ (four-body & -1.61 & -2.09 & -2.36 & -2.48 \\
term) & $(-1.43)$ & $(-1.89)$ & $(-1.56)$ & $(-1.97)$ \\
$E_{R}$ & 2.03 & 2.53 & 6.09 & 6.45 \\
$\Delta E$ & -134.07 & -180.47 & -253.51 & -295.06 \\
\hline \hline
\end{tabular}

as four-body terms form the attractive or additive part and contribute to a larger extent to total interaction energy as compared to the higher-body terms. The higher-body terms, $n>4$ body terms, are repulsive ones and destabilize the system constituting positive interaction energy. In general, the relaxation energy $\left(E_{R}\right)$ term forms a repulsive part of interaction energy $(\Delta E)$. This observation is in agreement with the earlier results on smaller water clusters. ${ }^{8}$

The results in Table II indicate that the two-body and three-body energy contributions per water molecule in the case of $\left(\mathrm{H}_{2} \mathrm{O}\right)_{n}, n=12,16$, and 20 increase gradually as $-8.89,-9.23$, and $-9.46 \mathrm{kcal} \mathrm{mol}^{-1}$, suggesting that the BSSE-uncorrected water $\cdots$ water interaction gradually increases with the cluster growth. However, this growth is too small numerically and such effects need to be examined further on several systems before general conclusions are made. The plot (cf. Fig. 2) of two-body energies against the number of molecules $(n)$ is linear in nature coupled with the large relative magnitude of this contribution. It suggests that the two-body terms contribute significantly for all cluster sizes.

Also, the variation of relaxation energy with clusters size (n) shows a stepwise increase (cf. Fig. 3). This trend may be taken as an indication of a gradual stabilization of larger clusters (say $n>40$ ), since the external molecules will suffer less strain as compared to the molecules inside the core.

BSSE corrections, ${ }^{12}$ are evaluated for $\left(\mathrm{H}_{2} \mathrm{O}\right)_{n}, n=12$, 16 , and 20 at RHF level of theory using $6-31 G(d, p)$, $6-31+G(d, p)$, and $6-311++G(2 d, 2 p)$ basis sets. The results in Table III show that the BSSE gets numerically reduced on the use of a higher-quality basis set. This is in agreement with the trends observed in the literature. ${ }^{25}$

TABLE VI. Comparison of MBAC energy analysis for acetamide (AM), formaldehyde (FLD), and acetaldehyde (ALD) with $\left(\mathrm{H}_{2} \mathrm{O}\right)_{16}$ at RHF/6-31G(d,p) level. Notations and other details as in Table I.

\begin{tabular}{lccc}
\hline \hline$E_{\text {Contr }}$ & $\mathrm{AM} \cdots\left(\mathrm{H}_{2} \mathrm{O}\right)_{16}$ & $\mathrm{FLD} \cdots\left(\mathrm{H}_{2} \mathrm{O}\right)_{16}$ & $\mathrm{ALD} \cdots\left(\mathrm{H}_{2} \mathrm{O}\right)_{16}$ \\
\hline$E$ (two-body term) & -158.11 & -154.45 & -154.02 \\
& $(-147.03)$ & $(-147.56)$ & $(-147.59)$ \\
$E$ (three-body term) & -23.18 & -21.87 & -21.80 \\
& $(-21.22)$ & $(-21.12)$ & $(-21.11)$ \\
$E$ (four-body term) & -2.07 & -1.76 & -1.77 \\
& $(-1.89)$ & $(-1.92)$ & $(-1.92)$ \\
$E_{R}$ & 2.68 & 2.26 & 2.27 \\
$\Delta E$ & -180.43 & -175.58 & -175.09 \\
\hline \hline
\end{tabular}

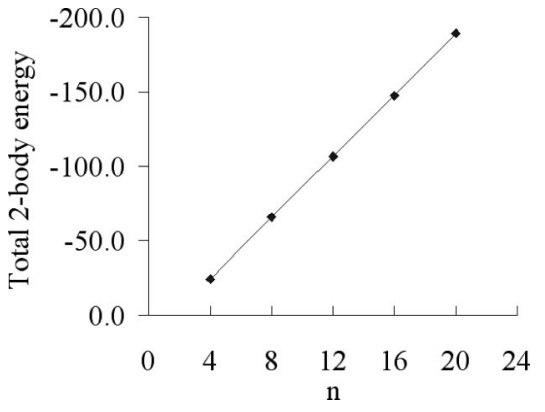

FIG. 2. Variation of total $E$ (two body), in $\mathrm{kcal} \mathrm{mol}^{-1}$, with cluster size (n), for water clusters.

It may be seen that the interactions observed in the weakly bound cluster are highly directional in nature. Further, the clusters studied in the present work are hydrogen bonded clusters and are known to be dominated by electrostatic interactions; simple electrostatic model calculations are performed on $\left(\mathrm{H}_{2} \mathrm{O}\right)_{n}, n=16$ and 20. The interaction energy $\Delta E_{\mathrm{el}}$ according to this model is $\Sigma q_{i} q_{j} / r_{i j}$, where $i \in A, j$ $\in B$ and $q_{i}, q_{j}$ are the electrostatic derived charges on the atoms of species $A$ and $B$ (generated by chelpg ${ }^{26}$ option through G94) and $r_{i j}$, the distance between them. It is seen from the plots of the number of positive and negative contributions to the $\Delta E_{\mathrm{el}}$ values and the number of minimum steps between the two water molecules within that clusters [cf. Figs. $7(\mathrm{a})-7(\mathrm{c})]$, that these interactions naturally decrease with distance. In the case of small clusters the molecules are most optimally oriented with respect to each other yielding favorable (negative) contribution arising from twobody and three-body interactions. The long-range randomness in orientation of water molecules is seen to increase on cluster growth. This fact leads to energetically smaller terms involving more steps for large clusters and cancellation of few favorable higher-body contributions, making the overall contributions to interaction energy due to the higher-body (four and more) terms repulsive. The plots in Figs. 7(a) and 7(b) indeed reveal this characteristic. It may be noted that the trends in the energies given by these model calculations for two-body terms match those of actual MBAC calculations on two-body terms [cf. Figs. 7(a) and 7(b)].

\section{Heteroclusters, $\mathrm{M} \cdot \cdots\left(\mathrm{H}_{2} \mathrm{O}\right)_{n}$}

MBAC program has also been applied to the most favorable structures of formamide *water heteroclusters,

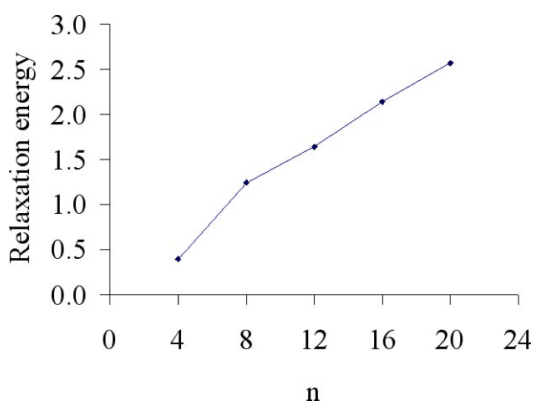

FIG. 3. Variation of relaxation energy $\left(E_{R}\right)$, in $\mathrm{kcal} \mathrm{mol}^{-1}$, with cluster size (n), for water clusters. 


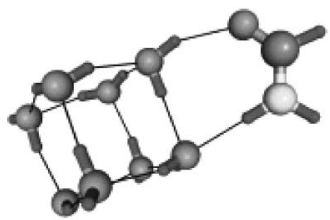

FM8

$\Delta \mathrm{E}(\mathrm{RHF})=-87.49$

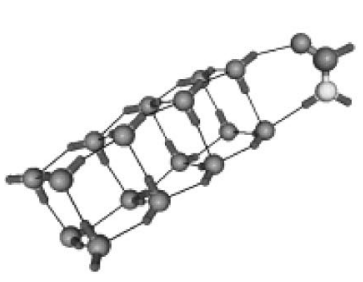

FM16 $\Delta \mathrm{E}(\mathrm{RHF})=-180.52$

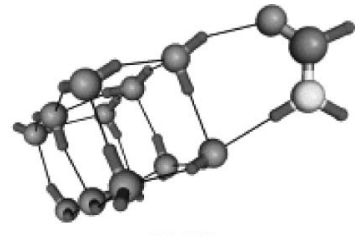

FM12

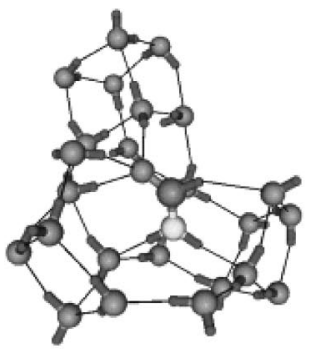

FM24 $\Delta E(R H F)=-253.48$ $\Delta E(R H F)=-134.11$

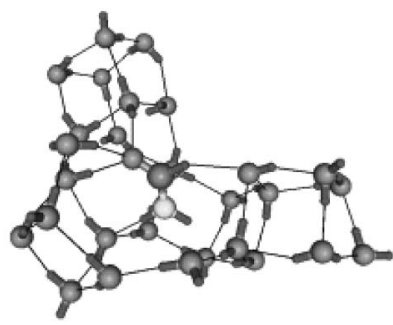

FM28

$\Delta \mathrm{E}(\mathrm{RHF})=-295.07$

FIG. 4. RHF/6-31G(d,p) optimized geometries (Ref. 24) of $\mathrm{HCONH}_{2} \cdots\left(\mathrm{H}_{2} \mathrm{O}\right)_{n}, n=8,12,16,24$, and 28 along with the interaction energies (in kcal mol${ }^{-1}$ ). See text for details.

FM $\cdots\left(\mathrm{H}_{2} \mathrm{O}\right)_{n}, n=12,16,24$, and 28 (cf. Fig. 4) at RHF/6-31G(d,p) levels. The details of MBA results are given in Table VI. Also, in order to find out the difference in binding abilities of amides and aldehydes, the analysis is also done for the prototype hydrated clusters of AM, FLD, and ALD with 16 water molecules (cf. Table VI).

The variations of total two-body contribution as well as relaxation energy $\left(E_{R}\right)$ with cluster size $(n)$ are studied for FM $\cdots\left(\mathrm{H}_{2} \mathrm{O}\right)_{n}$ clusters (cf. Fig. 5 and Table V). The slope of the line is seen to change linearly in the steps between $n$ $=8$ to 24 region. It is further seen that for the large cluster size, the contribution due to two-body terms still forms $85 \%-90 \%$ of the total interaction energy.

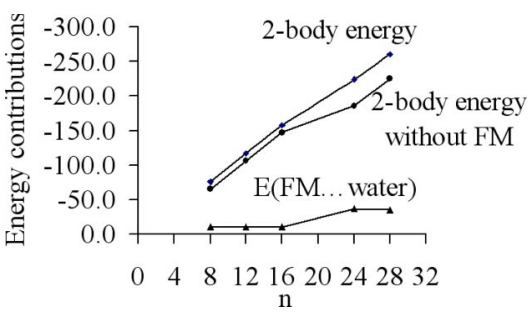

FIG. 5. Variation of $E$ (two-body), in $\mathrm{kcal} \mathrm{mol}^{-1}$, with cluster size (n) for $\mathrm{FM} \cdots\left(\mathrm{H}_{2} \mathrm{O}\right)_{n}$.

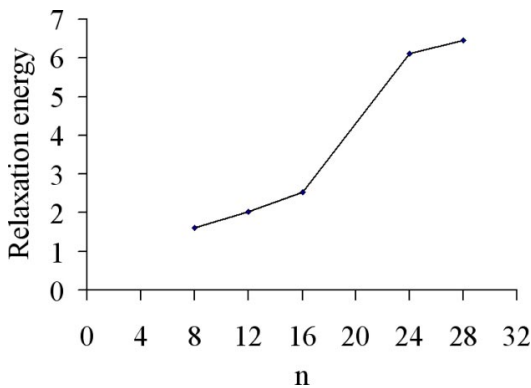

FIG. 6. Variation of $E_{R}$, in $\mathrm{kcal} \mathrm{mol}^{-1}$, with cluster size $(n)$ for FM $\cdots\left(\mathrm{H}_{2} \mathrm{O}\right)_{n}$.

The total two-body water $\cdots$ water interaction energy (cf. Fig. 5) is, however, numerically linear. However, the variation of relaxation energy $\left(E_{R}\right)$ with the cluster size shows an increase in stepwise manner (Fig. 6). Total FM $\cdots \mathrm{H}_{2} \mathrm{O}$ twobody interaction shows a jump from $\sim 11$ to $38 \mathrm{kcal} \mathrm{mol}^{-1}$ on going from $n=16-24$ (Fig. 6). This observation suggests that a part of the hydrated cluster is still not forming an envelope around the heteromolecule (formamide) and there is still some part of the cluster where further added water molecules can establish direct contact with the formamide molecule. The two-body contribution involving FM moiety shows initial stabilization (up to $n=16$ ). In the case of FM $\cdots\left(\mathrm{H}_{2} \mathrm{O}\right)_{n}, n=8,12$, and 16 , there are only two water molecules in contact with FM.

The added water molecules in the expanding clusters $(n$ increasing from 8 to 16) show very little interaction with FM. However, for FM24 and FM28, there are few more water molecules in contact with FM leading to an increase in FM $\cdots \mathrm{H}_{2} \mathrm{O}$ interactions and total two-body interactions as well.

It may be seen that the two-body energy contribution in case of amides (cf. Tables V and VI) is $\sim-158 \mathrm{kcal} \mathrm{mol}^{-1}$, whereas in the case of aldehydes it is $\sim-154 \mathrm{kcal} \mathrm{mol}^{-1}$ (Table V), hence the substrate $\cdots \mathrm{H}_{2} \mathrm{O}$ interaction [E(two body) $-E$ (two body excluding reference)] in the case of amides is stronger than the corresponding aldehydes by $\sim 3-4 \mathrm{kcal} \mathrm{mol}^{-1}$. This is the most striking observation coming from the energetics of two-body terms containing amide $\cdots \mathrm{H}_{2} \mathrm{O}$ and aldehyde $\cdots \mathrm{H}_{2} \mathrm{O}$ interactions.

The total two-body $\mathrm{H}_{2} \mathrm{O} \cdots \mathrm{H}_{2} \mathrm{O}$ interaction energy for all the $16 \mathrm{H}_{2} \mathrm{O}$ containing clusters is $\sim-147 \mathrm{kcal} \mathrm{mol}^{-1}$. Thus none of these substrates is seen to strengthen the $\mathrm{H}_{2} \mathrm{O} \cdots \mathrm{H}_{2} \mathrm{O}$ interactions. The proportions of energy contributions observed for FM $\cdots\left(\mathrm{H}_{2} \mathrm{O}\right)_{n}$ clusters are similar to those observed for the water clusters where the major contribution to $\Delta E$ comes from the two-, three-, and four-body energy terms and the higher-body (greater than four) contributions are very small. It is worthwhile to mention that the meaning of the terms structuring and destructuring effects in hydrated clusters is not yet clear and needs to be explored further.

It may be noted that in the case of heteroclusters also the electrostatic model calculations [Figs. 7(c) and 7(d)] are able 


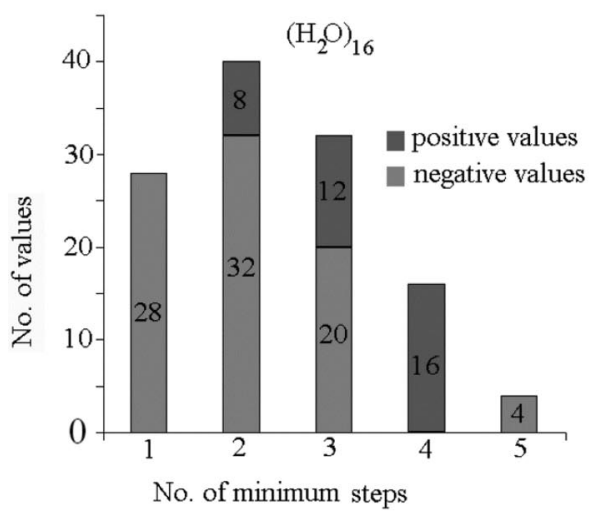

(a)

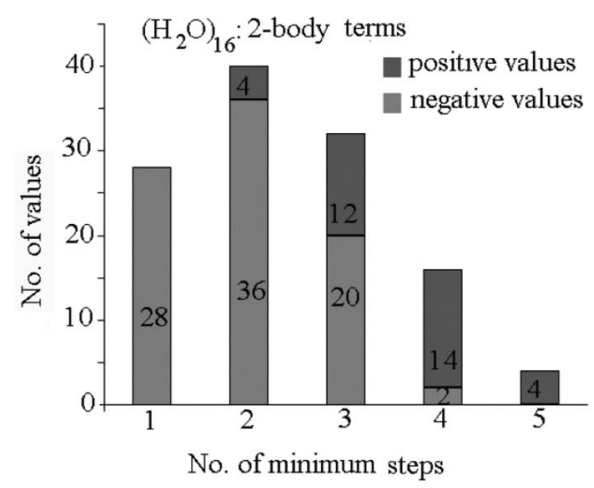

(b)

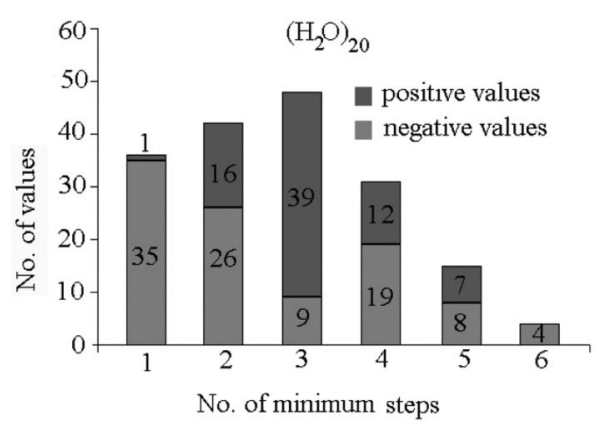

(c)

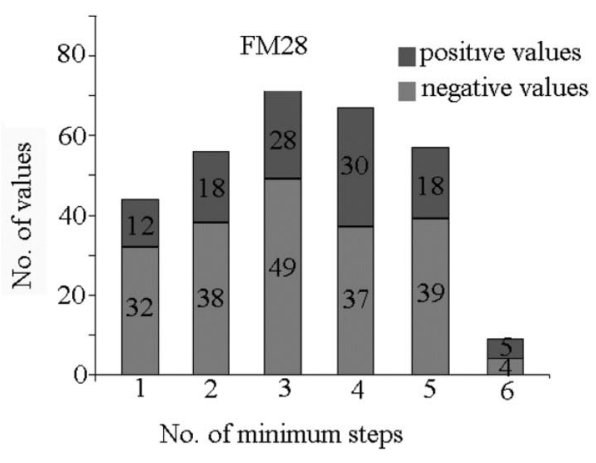

(d)
FIG. 7. Distribution of two-body terms for various clusters (a) $\left(\mathrm{H}_{2} \mathrm{O}\right)_{16}$ by model electrostatic calculations, (b) $\left(\mathrm{H}_{2} \mathrm{O}\right)_{16}$ by actual MBAC calculations, (c) model electrostatic calculations for $\left(\mathrm{H}_{2} \mathrm{O}\right)_{20}$, and (d) model electrostatic calculations for FM28. to offer a physical picture of nonfavorable and numerically small contributions arising due to higher-body terms.

\section{CONCLUDING REMARKS}

Understanding the many-body effects in molecular clusters is an important step for obtaining insights in the energetics and binding patterns. The present MBAC approach is capable of performing MBA with or without with BSSE corrections for weakly interacting homoclusters as well as heteroclusters of any size, in an efficient manner. The MBAC algorithm enables computation of up to $R$-body terms $(R$ $<N$ ) for an $N$-body cluster at any level of theory, viz., HF, MP2, and DFT using all the basis set available in popular $a b$ initio packages. ${ }^{19,20}$ Also, the approach for constructing the DM's of smaller clusters from the $N$-body one and also the systematic elimination of energetically insignificant higherbody terms implemented in the algorithm boosts its performance by a factor of $\sim 1.5-2.0$ over the conventional approach for large molecular clusters.

From the variety of examples presented in this paper, the typical contribution of two- and three-body terms is seen to be more than $95 \%$. For the larger water clusters, $\left(\mathrm{H}_{2} \mathrm{O}\right)_{n}$, $n>8$, this contribution turns out to be more than $98 \%$. The contribution due to nonadditive terms (terms generally fiveand higher-body ones) to the interaction energy decreases with increase in cluster size. In general, it has been noted that the percentage contribution of four-body energy component to interaction energy is $\sim 1.5 \%$, whereas the contribution due to all higher bodies $(>4)$ is generally nonfavorable. The electrostatic model calculations throw light on this fact, which may be attributed to the randomness in long-range molecular orientations. Also, the gradual increase in $\mathrm{H}_{2} \mathrm{O} \cdot \mathrm{H}_{2} \mathrm{O}$ interactions in water cluster on clustering indeed shows the ability of the MBAC approach in understanding the nature of binding and the cooperativity of interactions in molecular clusters. Also, total three-body energy contribution turns out to be typically $15 \%$ of the corresponding total two-body contribution.

In the case of $\left(\mathrm{H}_{2} \mathrm{O}\right)_{n}$, homoclusters, gradual increase in the total two-body term is found to be very small and warrants further study at higher levels of theory, better basis sets, and BSSE correction. Also, for homostructures as well as heteroclusters, the addition of more molecules at larger size (say $n>30$ ) may lead to the cluster growth without introducing appreciable strain in the overall cluster arrangement (Figs. 1 and 4). It may also be noted that the MBAC study can successfully differentiate the binding abilities of amides and aldehydes with water, by bringing out the stronger binding in the case of amides (FM). It has also been ascertained that the use of higher basis set can reduce the BSSE correction in supermolecular calculations to a reasonable extent and the MBAC algorithm provides an efficient tool for computing BSSE in large molecular aggregates.

With the advancement in computing architecture, efficient software, and growing interest in supramolecular assemblies, routine $a b$ initio treatment of homoclusters as well 
as heteroclusters containing $\sim 100$ molecules seems readily feasible in the coming decade. The MBAC approach developed in the present work offers a possibility of obtaining insights into the many-body effects of such large molecular aggregates in an efficient way.

\section{ACKNOWLEDGMENTS}

We are indebted to Dr. Rajeev K. Pathak, Dr. Sudhir A. Kulkarni, and Dr. K. Babu for useful discussions. Financial support from the Council of Scientific and Industrial Research (CSIR), New Delhi, India and the Center for Development of Advanced Computing (C-DAC), Pune, India, is gratefully acknowledged.

${ }^{1}$ M. J. Elrodt and R. J. Saykally, Chem. Rev. 94, 1975 (1994), and the references therein for detailed historical review.

${ }^{2}$ B. M. Axilrod and E. Teller, J. Chem. Phys. 11, 299 (1943).

${ }^{3}$ P. Rosen, J. Chem. Phys. 21, 1007 (1953).

${ }^{4}$ A. Shostak, J. Chem. Phys. 23, 1808 (1955).

${ }^{5}$ L. Jansen, Phys. Rev. 135, 1292 (1964).

${ }^{6}$ E. Lombardi and L. Jansen, Phys. Rev. 167, 822 (1968).

${ }^{7}$ D. Hankins, J. W. Moskowitz, and F. H. Stillinger, J. Chem. Phys. 53, 4544 (1970)

${ }^{8}$ S. S. Xantheas, J. Chem. Phys. 100, 7523 (1994)

${ }^{9}$ S. S. Xantheas, J. Chem. Phys. 104, 8821 (1996), and references therein.

${ }^{10}$ D. Tzeli, A. Mavridis, and S. S. Xantheas, J. Phys. Chem. A 106, 11327 (2002), and the references therein.
${ }^{11}$ S. A. Kulkarni, L. J. Bartolotti, and R. K. Pathak, Chem. Phys. Lett. 372, 620 (2003).

${ }^{12}$ S. F. Boys and F. Bernardi, Mol. Phys. 19, 553 (1970).

${ }^{13}$ J. C. White and E. R. Davidson, J. Chem. Phys. 93, 8029 (1990).

${ }^{14}$ H. Umeyama and K. Morokuma, J. Am. Chem. Soc. 99, 1316 (1990).

${ }^{15}$ P. Valiron and I. Mayer, Chem. Phys. Lett. 275, 46 (1997).

${ }^{16}$ K. Mierzwicki and L. Latjka, Chem. Phys. Lett. 380, 654 (2003).

${ }^{17}$ K. Babu and S. R. Gadre, J. Comput. Chem. 24, 484 (2003), and references therein; K. Babu, V. Ganesh, and S. R. Gadre, Theor. Chem. Acc. 111, 255 (2004).

${ }^{18}$ (a) GNU: http://www.gnu.org; (b) Intel Corporation, USA; cf. http:// www.intel.com for further details.

${ }^{19}$ M. J. Frisch, G. W. Trucks, H. B. Schlegel et al., GAussian 94, Revision B.3 Gaussian, Inc., Pittsburgh, PA, 1995.

${ }^{20}$ M. W. Schmidt, K. K. Baldridge, J. A. Boatz et al., J. Comput. Chem. 14, 1347 (1993); A. A. Granovsky, http://classic.chem.msu.su/gran/gamess/ index.html

${ }^{21}$ R. McWeeny, Rev. Mod. Phys. 126, 1028 (1962).

${ }^{22}$ G. A. Korn and T. M. Korn, Mathematical Handbook for Scientists and Engineers (McGraw-Hill, New York, 1961), Sec. 21.5.

${ }^{23}$ S. Maheshwary, N. Patel, N. Sathyamurthy, A. D. Kulkarni, and S. R. Gadre, J. Phys. Chem. A 105, 10525 (2001), cf. http://wwwwales.ch.cam.ac.uk/ wales/CCD/anant-watcl.html, a web-site related to the latest $a b$ initio investigations of water clusters.

${ }^{24}$ A. D. Kulkarni, K. Babu, S. R. Gadre, and L. J. Bartolotti, J. Phys. Chem. A 108, 2492 (2004).

${ }^{25} \mathrm{~S}$. Scheiner, Hydrogen Bond (Oxford University Press, New York, 1997).

${ }^{26}$ C. M. Breneman and K. B. Wiberg, J. Comput. Chem. 11, 361 (1990). 